\title{
Dissociation of intermolecular linkages of the sperm head and tail by primary amines, aldehydes, sulphydryl reagents and detergents
}

\author{
R. J. Young* and G. W. Cooper† \\ Reproductive Biology Unit, Department of Obstetrics and Gynecology, Cornell University \\ Medical College, 1300 York Avenue, New York 10021, U.S.A.
}

\begin{abstract}
Summary. Primary amines, pyridoxal and thiols induced separation of the mammalian sperm head and tail at specific sites across the head-tail junction. Primary amines and pyridoxal induced head detachment by allowing separation of the inner and outer nuclear envelope membranes adjacent to the tail basal plates. This detachment was prevented by prior reduction with sodium cyanoborohydride. The chemistry of amineinduced head separation and the similar action of pyridoxal indicate that the head and tail are joined by Schiff bases formed between proteins within the nuclear membranes. Head detachment with thiols occurred at two sites: across the connecting filaments linking the basal plate and the capitulum of the tail-neck complex and between the inner nuclear membrane and the nuclear chromatin. Mammalian epididymal spermatozoa exhibited species differences in susceptibility to head detachment induced by hydromechanical shear. The heads of mouse epididymal spermatozoa readily separated from the tails during vortexing whereas those from the vas deferens were resistant to shear. Head separation occurred at the same site as induced by primary amines. Rabbit spermatozoa from all parts of the epididymis were resistant to mechanical shear. Species differences in the mechanical stability of the head-tail junction suggest that the intermolecular Schiff bases linking the head and tail can be formed before or during sperm transport in the epididymis and that their formation probably occurs after the appearance of the periodic structures which bridge the inner and outer membranes of the nucleus in the region of the tail basal plate.
\end{abstract}

\section{Introduction}

The mammalian spermatozoon is a highly differentiated cell whose structural features have been extensively investigated at the ultrastructural level. Although a role in the fertilization process has been recognized for some of the sperm organelles, e.g. the acrosome and mitochondria, at present there is limited knowledge of the molecular components and organization of most sperm structures. Further advances in such analysis require the development of methods for the isolation of particular organelles and structural components of the sperm head and tail. A first step in this endeavour is the separation of the sperm head and tail. Methods developed for such detachment have included sonication, pressure cavitation, use of detergents, thiol reagents, extremes of $\mathrm{pH}$ and proteolytic enzymes (Calvin, 1976; Balhorn, Gledhill \& Wyrobeck, 1977; Brotherton, 1977). The rather severe conditions and non-specific methods used have precluded the preparation of

* Present address: School for Biomedical Education, The City College, 138th Street \& Convent Avenue, New York, New York 10031, U.S.A.

$\dagger$ Present address: Fertility Laboratory, North Shore University Hospital, Department of Obstetrics and Gynecology, 300 Community Drive, Manhasset, Long Island, New York 11030, U.S.A. 
undamaged heads or tails and of heads which do not contain remnants of tail structures. Moreover, none of the above methods gives information about the chemical nature of the head-tail junction. This report describes a method for the preparation of mammalian sperm heads free of tail structures using primary amines. The intermolecular connections across the mammalian spermatozoon's head-tail junction were studied by using primary amines, thiols, aldehydes and amino-thiols to effect head-tail separation. The relation between mechanical stability to vortexing and the action of primary amines in effecting head-tail separation were also investigated.

\section{Materials and Methods}

Spermatozoa. Rabbit spermatozoa collected into an artificial vagina (First, 1971) from fertile New Zealand White bucks were separated from seminal plasma and prostatic vesicular bodies by centrifugation for $3 \mathrm{~min}$ at $10000 \mathrm{~g}$, followed by resuspension in SET buffer, $\mathrm{pH} 7 \cdot 2(0 \cdot 1 \mathrm{M}$-Tris$\mathrm{HCl}, 0.15 \mathrm{M}-\mathrm{NaCl}, 0.005 \mathrm{M}-\mathrm{EDTA}$ ) (Young, 1979a). Sperm suspensions (1-3 $\times 10^{8}$ in $3 \mathrm{ml}$ ) were centrifuged through $2.05 \mathrm{M}$-sucrose in SET buffer $(10 \mathrm{ml})$ for $60-75 \mathrm{~min}$ at $107000 \mathrm{~g}$. The walls of the centrifuge tubes were wiped with tissues (Kimwipes) moistened with SET buffer to prevent contamination with cellular debris and the contents of detached sperm acrosomes (Young, 1979a). These pellets were then resuspended in SET buffer and recentrifuged through $2.05 \mathrm{M}$-sucrose to remove the last traces of seminal and acrosomal enzymes. Sucrose was removed by washing with SET buffer. Human semen, collected by masturbation, was centrifuged for 3 min at $2000 \mathrm{~g}$ after liquefaction and spermatozoa were washed twice by centrifugation in SET buffer. Rabbit, mouse, rat, hamster (Mesocricetus auratus), degu (Octodon degus) and guinea-pig spermatozoa were released from epididymides and vasa deferentia into Medium 199 (Grand Island Biological Co., New York) and washed once by centrifugation in SET buffer. Rhesus monkey (Macaca mulatta) spermatozoa obtained by electroejaculation (courtesy of Dr K. Sundaram, Population Council, New York) were washed as above for human spermatozoa.

Head-tail separation. Reagents used were of the highest purity available, and amines were not distilled unless highly coloured. Amines were added to sperm suspensions $\left(0 \cdot 5-5 \times 10^{7} / \mathrm{ml}\right)$ in SET buffer to induce head-tail detachment. These mixtures, in $10 \times 75 \mathrm{~mm}$ test tubes, were immediately vortexed for 15-30 sec (Vari-Whirl: VWR Scientific Co., New York) at maximum setting. Head-tail detachment after each treatment was assessed under Zeiss phase-contrast optics ( $\times$ 250-400). Percentages of head-tail detachments were calculated from the mean of triplicate random counts of 100 sperm heads and intact spermatozoa, minus the mean percentage background detachment obtained after vortexing in the absence of added reagent. Reaction mixtures were incubated at $37^{\circ} \mathrm{C}$ for up to $6 \mathrm{~h}$ before vortexing for those amines that gave no or low percentages of head-tail detachment. The actions of other reagents were assessed similarly or after incubations for up to $16 \mathrm{~h}$ at $37^{\circ} \mathrm{C}$.

Reduction with sodium cyanoborohydride (Aldrich Chemical Co., Milwaukee, Wisconsin) was carried out by adding $\mathrm{NaCNBH}_{3}$ to sperm suspensions $\left(0 \cdot 5-1 \times 10^{7}\right)$ in $1 \mathrm{ml} \mathrm{SEC} \mathrm{buffer}(0 \cdot 1 \mathrm{M}-$ citrate, $\mathrm{pH} 5,0.15 \mathrm{M}-\mathrm{NaCl}, 0.005 \mathrm{M}-\mathrm{EDTA})$. After $1 \mathrm{~h}$ incubation at $22^{\circ} \mathrm{C}$, spermatozoa were centrifuged $5 \mathrm{~min}$ at $10000 \mathrm{~g}$ and washed twice with SET buffer before testing the ability of amines to dissociate the sperm head-tail junction.

Preparative isolation of rabbit sperm heads and tails. Spermatozoa were purified by centrifugation through $2.05 \mathrm{M}$-sucrose (see above). Heads and tails were detached by mixing with $n$-butylamine ( 1 $\mu \mathrm{l} / 10^{6}$ spermatozoa and vortexing for $15-30 \mathrm{sec}$ at maximum setting on a Vari-Whirl mixer. This was repeated until head detachment was complete. Detached heads and tails were separated by centrifugation through a discontinuous gradient of $2.05 \mathrm{M}$ - and $2.5 \mathrm{M}$-sucrose in SET buffer (5-6 ml each) for 30-60 min at $82000 \mathrm{~g}$. Detached tails were found at the SET buffer-2.05 $\mathrm{M}$-sucrose interface and in the $2.05 \mathrm{M}$-sucrose layer, whereas intact spermatozoa were found in the $2.5 \mathrm{M}$ sucrose. The pellets consisted primarily of detached sperm heads with minor numbers of intact 
sperm and isolated tails. Pellets were resuspended and washed in SET buffer to remove sucrose. Separated heads tended to aggregate and to stick to glass, but such losses could be reduced by using polyethylene tubes or siliconized glassware, and particularly by using only the minimal amount of $n$-butylamine needed to induce head detachment. Aggregation was also prevented by adjusting the $\mathrm{pH}$ of butylamine-treated sperm suspensions to $\mathrm{pH} 7.5$ with $2 \mathrm{~N}-\mathrm{HCl}$ and by adding bovine serum albumin (final conc. $0 \cdot 25 \%$ ) before discontinuous sucrose centrifugation (M. Berrios, personal communication). Sperm head recovery was $70-90 \%$. Contamination with intact spermatozoa or tails was $\leq 1 \%$ after a second discontinuous sucrose gradient centrifugation.

Electron microscopy. Electron microscopy was carried out on spermatozoa fixed in $4 \%$ glutaraldehyde in $0.1 \mathrm{M}$-phosphate buffer $(\mathrm{pH} \mathrm{7.4)}$ ) for $1-2 \mathrm{~h}$, followed by a $1 \mathrm{~h}$ post-fixation in $1 \%$ $\mathrm{OsO}_{4}$ in $0.1 \mathrm{M}$-phosphate buffer. Sperm pellets washed in phosphate buffer were dehydrated in a graded ethanol series to absolute ethanol, to propylene oxide followed by embedding in Epon 812 . Thin sections cut on diamond knives were stained with lead citrate and uranyl acetate (in $10 \%$ ethanol) and observed with a Jeol 100B electron microscope at $80 \mathrm{kV}$. Replicas of spermatozoa after primary amine treatment were prepared by fixing washed amine-treated spermatozoa to polylysine-coated glass cover-slips with $1 \%$ glutaraldehyde in $0 \cdot 1 \mathrm{M}$-phosphate buffer. Specimens critical-point dried with liquid $\mathrm{CO}_{2}$ were rotary-shadowed with platinum-carbon in a Balzers high vacuum evaporator followed by detachment from glass with hydrofluric acid and treatment with sodium hypochlorite (Clorox: Clorox Co., Oakland, California, U.S.A.).

\section{Results}

\section{Head-tail separation by amines, thiols and aldehydes}

The head-tail junction of freshly ejaculated rabbit spermatozoa was resistant to shear forces generated by vortexing. In contrast, $90-95 \%$ of heads separated from the tails when saline-washed sperm suspensions were vortexed for $15-30 \mathrm{sec}$ after addition of $n$-butylamine (Cooper \& Young, 1977; Young \& Cooper, 1979). Quantitative assays of the effects of selected amines, as well as

Table 1. Head-tail detachment of rabbit spermatozoa ${ }^{a}$

\begin{tabular}{|c|c|c|c|}
\hline Compound & $\begin{array}{c}\text { Range of } \\
\text { detachment } \\
(\%)\end{array}$ & Compound & $\begin{array}{c}\text { Range of } \\
\text { detachment } \\
(\%)\end{array}$ \\
\hline Ethylamine & $95-98$ & $n$-Butyl alcohol & $\mathbf{0}$ \\
\hline Ethylenediamine & $87-92$ & Ethyl alcohol & $\mathbf{0}$ \\
\hline$n$-Butylamine & $87-96$ & iso-Butyric acid & 0 \\
\hline iso-Butylamine & $79-85$ & Pyridoxal hydrochloride ${ }^{\mathrm{e}}$ & $66-73$ \\
\hline tert-Butylamine & $82-85$ & Benzaldehyde ${ }^{f}$ & 0 \\
\hline Cyclohexylamine & $58-68$ & Acetaldehyde $f$ & 0 \\
\hline Penicillamine $^{b}$ & $18-37$ & Glyceraldehyde $^{f}$ & 3 \\
\hline Ethanolamine & $18-30$ & Dithiothreitolg & $65-85$ \\
\hline Amylamine ${ }^{d}$ & $16-20$ & Thioglycolic acid ${ }^{\mathrm{h}}$ & $68-80$ \\
\hline Benzylamine $^{d}$ & 5 & $\beta$-Mercaptoethanol ${ }^{i}$ & $80-85$ \\
\hline 3,4-Dimethoxyphenethylamine & $1-3$ & Sodium dodecyl sulphatej & 100 \\
\hline Di-n-Butylamine & $1-3$ & Sarkosyl ${ }^{k}$ & $85-92$ \\
\hline Di- $n$-Pentylamine & $1-3$ & Cetyltrimethylammonium bromide ${ }^{l}$ & 0 \\
\hline Tri-n-Butylamine & $1-3$ & $\mathrm{HCl}-\mathrm{KCl}$ buffer, $\mathrm{pH} 2 \cdot 2^{\mathrm{m}}$ & $10-12$ \\
\hline$n$-Butylacetamide & 0 & $\mathrm{NaOH}-\mathrm{NaCl}$ buffer, $\mathrm{pH} 12 \cdot 5^{\mathrm{n}}$ & 90 \\
\hline
\end{tabular}

(a) Ejaculated spermatozoa ( $1-5 \times 10^{7}$ ), washed by centrifugation through $2.05 \mathrm{M}$-sucrose, were mixed in $1 \mathrm{ml} \mathrm{SET}$ buffer with each reagent (0.2-0.5 mM) and vortexed for 15-30 sec. Ranges of head-tail dissociation are based on triplicate counts of intact and dissociated spermatozoa minus background dissociations after vortexing in SET buffer alone for $30 \mathrm{sec}$. (b) $0.6 \mathrm{mM}, 37^{\circ} \mathrm{C}, 2 \mathrm{~h}$; (c) $0.7 \mathrm{mM}, 37^{\circ} \mathrm{C}, 16 \mathrm{~h}$; (d) $37^{\circ} \mathrm{C}, 16 \mathrm{~h}$; (e) $0.15 \mathrm{~mm}, 37^{\circ} \mathrm{C}, 2 \mathrm{~h}$; (f) $37^{\circ} \mathrm{C}, \mathrm{Tris}-\mathrm{HCl}$, pH $8,16 \mathrm{~h}$; (g) $0.01 \mathrm{mM}, 37^{\circ} \mathrm{C}, 1 \mathrm{~h}$; (h) $0.1 \mathrm{mM}, 37^{\circ} \mathrm{C}, 3 \mathrm{~h}$; (i) $0.14 \mathrm{mM}, 37^{\circ} \mathrm{C}, 1 \mathrm{~h}$; (j) $0.2 \%$; (k) $2.5 \%, 37^{\circ} \mathrm{C}, 5 \mathrm{~min}$; (l) $1.0 \% ;(\mathrm{m}) 37^{\circ} \mathrm{C}, 40 \mathrm{~min} ;(\mathrm{n}) 22^{\circ} \mathrm{C}, 5 \mathrm{~min}$. 
thiols, aldehydes, alcohols, acids, detergents, buffers of high and low $\mathrm{pH}$ and concentrated salt solutions were tested with ejaculated rabbit spermatozoa (Table 1). Some of these reagents required extended incubations at $37^{\circ} \mathrm{C}$ before any effect was noted. To eliminate the possible action of seminal and acrosomal enzymes on sperm structures during incubation, rabbit spermatozoa were centrifuged through $2.05 \mathrm{M}$-sucrose, a procedure which removes seminal plasma enzymes and acrosomal proteases (Young, 1979a, b) (Text-fig. 1). Text-figure 1 also outlines a modified procedure for the preparative isolation of rabbit sperm heads and tails after $n$-butylamine treatment. Addition of albumin and $\mathrm{pH}$ adjustment of amine-containing sperm suspensions, before discontinuous sucrose centrifugation, prevented the sperm head aggregation with consequent contamination of isolated heads with intact spermatozoa which occurs without these changes in our previous procedure (Young \& Cooper, 1979).

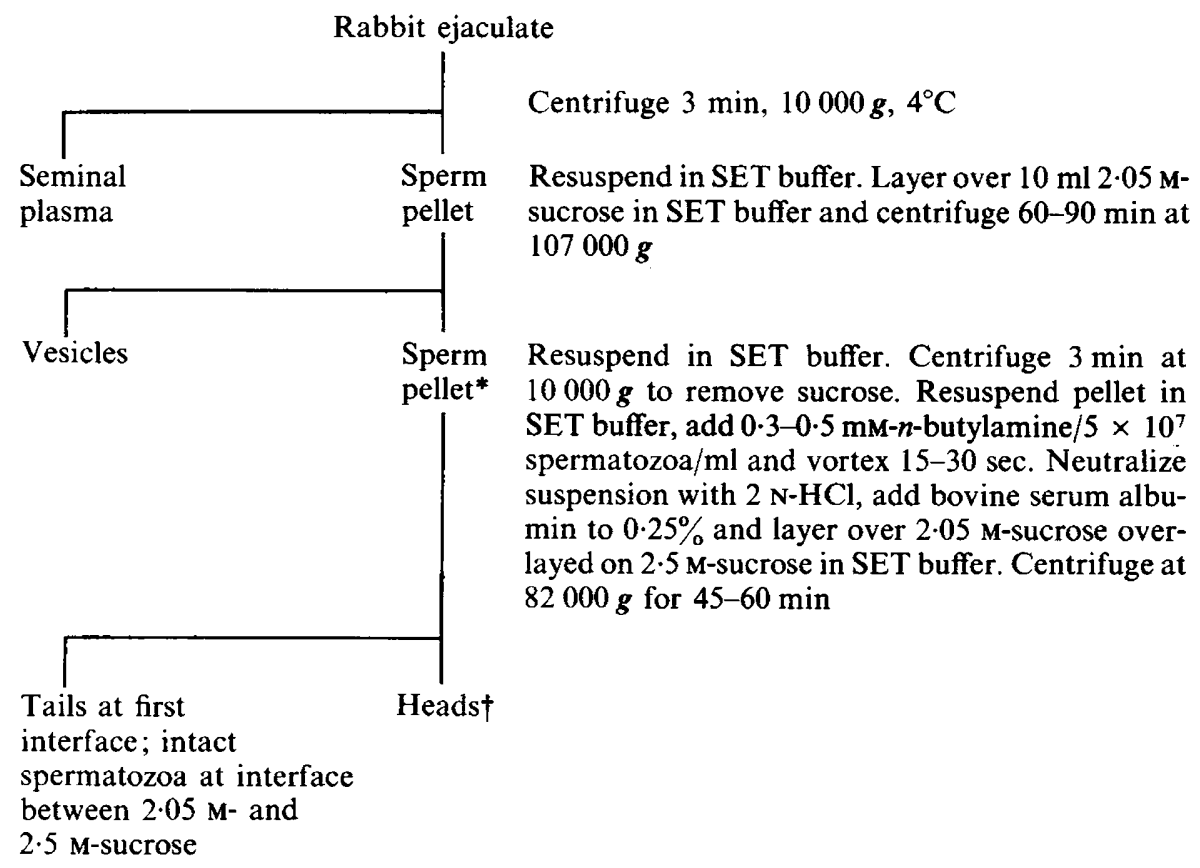

Text-fig. 1. Scheme for separation of sperm heads and tails. *Acrosomes are detached during this centrifugation (Young, 1979a). †Head aggregation and loss can be reduced by using the minimum amount of amine for head detachment, by neutralizing with $\mathrm{HCl}$ followed by addition of bovine serum albumin to $0.25 \%$, and by using plastic vessels or siliconized glassware. A second centrifugation through the discontinuous gradient reduced tail and intact sperm contamination to $1-2 \%$ with $70-90 \%$ overall yield of heads.

Primary amines, thiols, pyridoxal hydrochloride, anionic detergents and buffers of low and high $\mathrm{pH}$ were effective in separating the heads and tails of rabbit spermatozoa (Table 1). Quantitative head-tail cleavage was obtained after brief vortexing only with reactive primary amines. Anionic detergents, $1 \%$ sodium dodecyl sulphate or $2.5 \%$ sarkosyl $\left(5 \mathrm{~min}\right.$ at $\left.37^{\circ} \mathrm{C}\right)$, were equally effective. The cationic detergent cetyl trimethylammonium bromide failed to dissociate rabbit spermatozoa into heads and tails, in contrast to its effect on mouse spermatozoa (Balhorn et al., 1977). Sodium hydroxide-sodium chloride buffer, $\mathrm{pH} 12.5\left(5 \mathrm{~min}, 37^{\circ} \mathrm{C}\right)$ also induced $100 \%$ head-tail dissociation, but $<12 \%$ head detachment occurred after 40 min incubation at $37^{\circ} \mathrm{C}$ with an $\mathrm{HCl}-\mathrm{KCl}$ buffer, $\mathrm{pH}$ $2 \cdot 2$. A reactive aldehyde, pyridoxal hydrochloride, induced $73 \%$ head detachment after $2 \mathrm{~h}$ incubation at $37^{\circ} \mathrm{C}$, whereas less reactive aldehydes had no effect even after $16 \mathrm{~h}$ incubation. Concentrated solutions of $\mathrm{NaCl}(2 \mathrm{M})$, or $\mathrm{MgSO}_{4}(1 \mathrm{M})$ had no effect on head detachment. In 
contrast to the observation of Millette, Spear, Gall \& Edelman (1973), thiols alone induced head detachment of rabbit spermatozoa. Brief $(25-30 \mathrm{sec})$ vortexing after $1 \mathrm{~h}$ incubation at $37^{\circ} \mathrm{C}$ with $0.01 \mathrm{M}$-dithiothreitol or $0.14 \mathrm{M}-\beta$-mercaptoethanol in SET buffer or $0.1 \mathrm{M}$ Tris- $\mathrm{HCl}, \mathrm{pH} 8$, resulted in head-tail cleavage of $>80 \%$ of spermatozoa. Thioglycolic acid also induced head detachment, although a $3 \mathrm{~h}$ incubation was required for $80 \%$ detachment with $0 \cdot 1$ or $0.3 \mathrm{~m}$ solutions in SET buffer. Under these conditions decondensation of sperm head chromatin also occurred with thioglycolic acid (Pl. 1, Fig. 6). All thiol reagents produced degradation to various degrees of the neck connecting piece components and tail dense fibres, and disrupted the mitochondrial sheath. Isobutyric acid and butyl alcohol did not induce head-tail detachment.

A variety of mammalian spermatozoa were tested for their reactivity towards $n$-butylamine: high percentages of head-tail detachment were induced in ejaculated human and rhesus monkey spermatozoa and epididymal mouse, hamster, guinea-pig and degu spermatozoa. Mouse epididymal spermatozoa were further studied for their reactivity towards primary, secondary, and tertiary amines and thiols with results similar to those obtained with rabbit spermatozoa. The rat was a notable exception in that $n$-butylamine did not induce significant head detachment when cauda epididymal or vas spermatozoa were tested. Increasing the amount of $n$-butylamine caused rat spermatozoa to agglutinate without appreciable head-tail detachment.

\section{Sites of detachment}

Each class of reagent tested induced head-tail detachment at specific and limited sites across the head-tail axis. Electron microscopy of amine-treated spermatozoa (rabbit, human, mouse, guinea-pig and degu) showed that tail detachment during vortexing occurred exclusively at the level of the nuclear envelope membranes (Pl. 1, Fig. 1) (Cooper \& Young, 1977; Young \& Cooper, 1979). The inner membrane of the nuclear envelope remained intact and associated with the nuclear chromatin over the tail implantation fossa (Young \& Cooper, 1979), whereas the outer membrane of the envelope and basal plate(s) separated together where the basal plate(s) contacted the outer membrane of the nuclear envelope (Pl. 1, Fig. 2). Pyridoxal hydrochloride, an aldehyde, similarly induced head-tail separation (Table 1) across the nuclear envelope membranes of rabbit spermatozoa, the only species tested with this reagent.

Thiols induced head-tail separation at the level of the tail implantation fossa and/or across the fibrous strands which link the basal plate(s) to the capitulum of the neck connecting piece. Head detachment after dithiothreitol treatment (rabbit, mouse) occurred over the tail implantation fossa, leaving the nuclear chromatin devoid of membrane in this region (Pl. 1, Fig. 3). The nuclear envelope membranes separated as a unit and retained their association with the basal plate(s) (Pl. 1, Fig. 4). $\beta$-Mercaptoethanol treatments gave the same types of tail separations as dithiothreitol, but with greater percentages of spermatozoa exhibiting degradation of the connecting piece complex, including cleavage of the fibrous strands linking the basal plate(s) to the connecting piece. Decondensation of nuclear chromatin did not occur with either of these thiols, whereas thioglycolic acid induced chromatin dispersal as well as degradation of the neck connecting piece complex and outer dense fibres of the tail. Strands of dispersed nucleo-chromatin were associated with the inner membrane of the nuclear envelope along its entire inner surface. Rabbit spermatozoa treated with thioglycolic acid showed this association (P1. 1, Fig. 5) when fixed without vortexing. This treatment leaves the nuclear envelope intact and associated with the basal plates in the same manner as does dithiothreitol and mercaptoethanol treatment. The transverse electron-dense material which appears to bridge the inner and outer membranes of the nuclear envelope remained after thiol treatment (P1. 1, Fig. 6). Penicillamine treatment resulted in two types of head-tail separation consistent with the combined actions of the thiol and the amine of this bifunctional reagent.

Head-tail separation after detergent treatments (Table 1) was in accord with their membrane solubilizing properties and indicates that a continuous intermolecular linkage does not exist across 
the head-tail junction between the nuclear chromatin and the basal plates of the tail. High and low $\mathrm{pH}$ buffer solutions (Table 1) had the same effects on the ultrastructure of rabbit spermatozoa as reported for the mouse, i.e. at low but not high $\mathrm{pH}$ the basal plates were attached to the tail after separation (Millette et al., 1973).

\section{Nature of the head-tail linkage within the nuclear envelope}

The percentage of head-tail detachment induced by vortexing for $30 \mathrm{sec}$ after addition of a primary amine was dependent on the amount of amine added and on its reactivity but not on the species of spermatozoa (Tables $1 \& 2$ ). The amount of $n$-butylamine required to induce $90-100 \%$ head-tail detachment was determined by titration with buffer-washed mouse and rabbit spermatozoa. Mouse spermatozoa required $33.4 \mu \mathrm{mol} n$-butylamine $/ 10^{6}$ spermatozoa, whereas $65 \cdot 3$ $\mu \mathrm{mol}$ were required to exert the same effect on an equal number of rabbit spermatozoa. Primary amine-induced head-tail detachment was also pH dependent (Table 3). Maximum head-tail detachment occurred at $\mathrm{pH} 10 \cdot 5-11$, the $\mathrm{p} K_{\mathrm{a}}$ of the amines, and was low when the amino group was protonated at neutral or acid $\mathrm{pH}$.

The ability of only reactive aldehydes and primary amines to induce sperm head-tail detachment (Table 1) within the nuclear envelope (see P1. 1, Figs $2 \& 4$ ), the quantitative dependence of this (Table 2), and its pH dependency (Table 3), is consistent with the existence of Schiff-base cross-links at this site. The morphological observation that the basal plate remains with the sperm head under conditions of high but not low $\mathrm{pH}$ is also consistent with this conclusion as Schiff bases are stable to alkali but not acid. Schiff bases undergo transimination reactions with primary amines and displacement reactions with reactive aldehydes (Dayagi \& Dagani, 1970; Leussing, 1976), generating new Schiff bases with the added reagents. The head and tail of the spermatozoon thus appears to be joined by Schiff bases formed between protein molecules within the nuclear envelope at the implantation fossa. Reduction of Schiff bases to the secondary amine prevents transimination reactions and would be expected to block head-tail detachment. This was tested by

\section{PLATE 1}

Rabbit spermatozoa fixed in $4 \%$ glutaraldehyde in $0 \cdot 1 \mathrm{M}$-phosphate buffer after the various treatments indicated.

Fig. 1. Platinum-carbon replica of the posterior of a sperm head after tail dissociation with $n$ butylamine. Note the asymmetric curve of the concave tail implantation fossa (arrow). $\times 18400$.

Fig. 2. Neck complex of sperm tail dissociated from the head by $n$-butylamine treatment; frontal section through head-tail axis. Arrows point to the outer membrane of the nuclear envelope which remains associated with the tail basal plates. $\times 105000$.

Fig. 3. Implantation fossa of sperm head after dithiothreitol treatment; frontal section. Arrow points to nuclear chromatin devoid of nuclear envelope membranes. $\times 50000$.

Fig. 4. Neck complex of sperm tail separated from the head by dithiothreitol treatment, as in Fig. 3. The plane of section through the neck complex is perpendicular to that in Fig. 2. The arrow points to the inner membrane of the intact nuclear envelope remaining associated with the basal plates. $\times 105000$.

Figures 5 \& 6. Head-tail junctions of spermatozoa fixed without vortexing following treatment with thioglycolic acid; frontal sections.

Fig. 5. Chromatin strands (arrow) extend between the nuclear envelope and partly decondensed sperm nucleus. Note the extensive degradation of the neck complex and tail striated columns/dense fibres which occurs after thioglycolic acid treatment. $\times 50000$.

Fig. 6. Arrow on tail basal plate points to the nuclear envelope with transverse, periodic electron-dense lines bridging its inner and outer membranes. Strands of nuclear chromatin contact the inner membrane of the envelope. $\times 105000$. 


\section{PLATE 1}
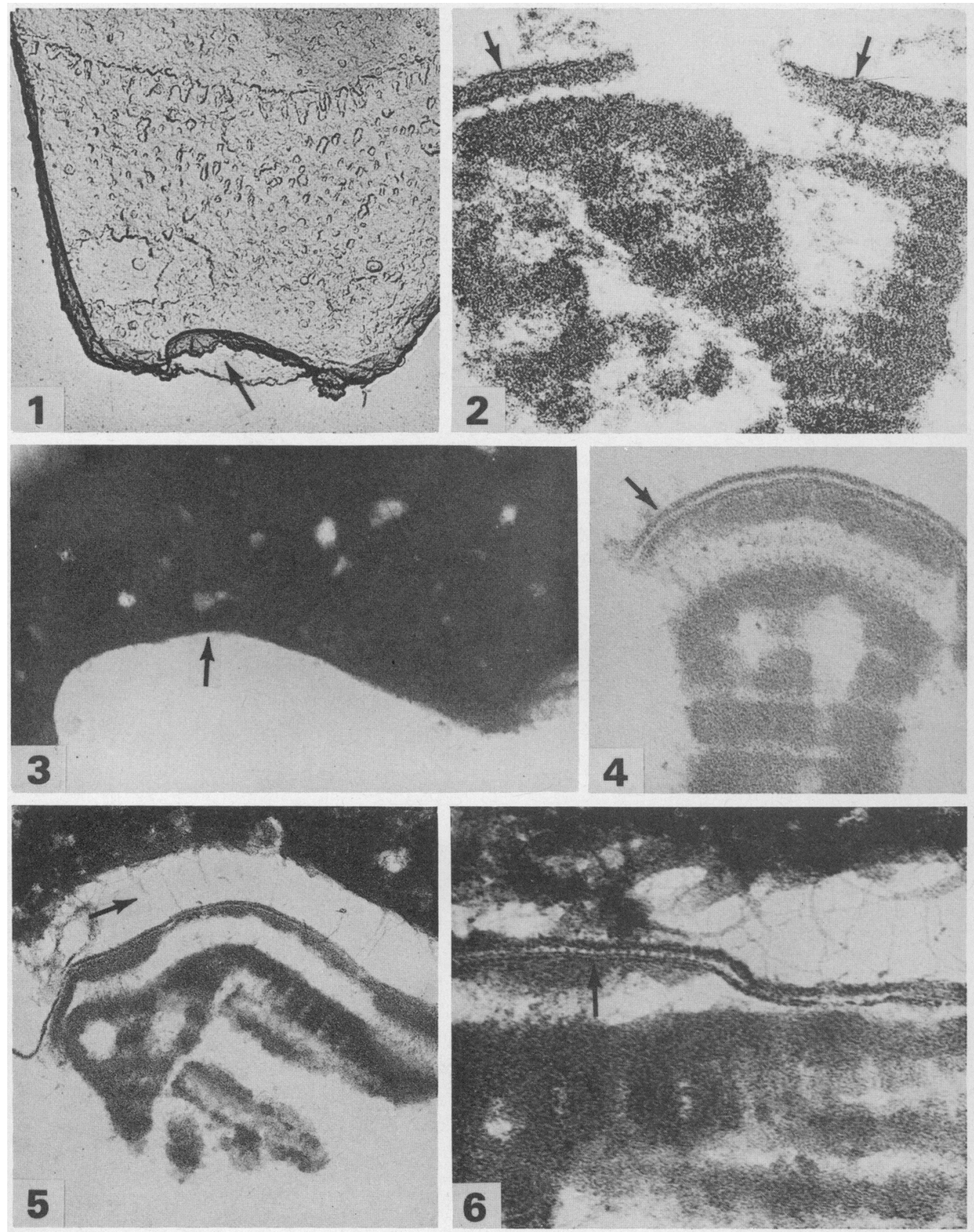

(Facing p. 6) 
Table 2. Dependence of head-tail detachment on amine addition

\begin{tabular}{cccc}
\hline Spermatozoa* & Amine & $\begin{array}{c}\text { Amount } \\
(\mu \mathrm{l})\end{array}$ & $\begin{array}{c}\text { Mean \% } \\
\text { detachment }\end{array}$ \\
\hline Mouse $\left(0 \cdot 5 \times 10^{6}\right)$ & tert-Butylamine & 5 & 25 \\
& & 10 & 75 \\
Mouse $\left(1 \times 10^{6}\right)$ & $n$-Butylamine & 10 & 70 \\
& & 15 & 90 \\
Rabbit $\left(3 \times 10^{6}\right)$ & Cyclohexylamine & 20 & 30 \\
& & 50 & 88 \\
& iso-Butylamine & 20 & 29 \\
& & 50 & 90 \\
& sec-Butylamine & 30 & 16 \\
& & 50 & 99 \\
\hline
\end{tabular}

* Mouse cauda epididymal and sucrose-washed ejaculated rabbit spermatozoa were vortexed for $15-30 \mathrm{sec}$ after addition of $\mu \mathrm{l}$ volumes of amine per $\mathrm{ml}$ SET buffer.

$\dagger$ Means of duplicate counts of intact and dissociated spermatozoa after subtraction of the mean background dissociations obtained by vortexing alone.

Table 3. Effect of $\mathrm{pH}$ on head-tail detachment induced by n-butylamine*

\begin{tabular}{lcc}
\hline \multicolumn{1}{c}{ Buffer } & $\begin{array}{c}\mathrm{pH} \text { after amine } \\
\text { addition } \dagger\end{array}$ & $\begin{array}{c}\% \text { Detachment } \\
\text { (range }\end{array}$ \\
\hline $0 \cdot 1 \mathrm{M}-$ Phosphate $(\mathrm{pH} \mathrm{10 \cdot 5)}$ & $10 \cdot 5-11$ & $86-88$ \\
$0 \cdot 1 \mathrm{M}-$ Carbonate $(\mathrm{pH} \mathrm{9 \cdot 0)}$ & $9-9 \cdot 5$ & $35-42$ \\
$1 \mathrm{M}-$ Tris-HCl (pH 7.2) & $7-8$ & $15-20$ \\
$1 \mathrm{M}-$ Citrate (pH 5.0) & $5-6$ & $5-10$ \\
\hline
\end{tabular}

* Sucrose-washed ejaculated rabbit spermatozoa $\left(1-3 \times 10^{7} / \mathrm{ml}\right.$ buffer $)$.

$\dagger 0 \cdot 1-0 \cdot 3 \mathrm{~mm}-n$-butylamine.

$¥$ Background detachments were determined in the buffer used for that experiment, and they ranged from 1 to $5 \%$ for all $\mathrm{pH}$ values.

studying the effect of primary amines on spermatozoa that had been reduced by sodium cyanoborohydride. The aldimine bond of a Schiff base is rapidly and selectively reduced under mild conditions by this reagent (Lane, 1975) to give a secondary amine. Ejaculated spermatozoa of rabbit and rhesus monkey were resistant to head detachment by $n$-butylamine after prior reduction with sodium cyanoborohydride (Table 4). Cauda epididymal spermatozoa of the hamster were similarly resistant. The mouse cauda epididymal and vas deferens spermatozoa showed a marked difference in behaviour after sodium cyanoborohydride reduction. Head separations of cauda epididymal spermatozoa were lowered by only $12-28 \%$ after reduction. Cauda spermatozoa formed aggregates in SEC buffer, pH 5, and also appeared to be structurally unstable, because high background head detachments were obtained with vortexing alone. In contrast, vas deferens spermatozoa were stable in SEC buffer, $\mathrm{pH} 5$, did not aggregate at this $\mathrm{pH}$ and were resistant to the action of $n$-butylamine after reduction with cyanoborohydride (Table 4).

\section{Head detachment of epididymal spermatozoa}

Rabbit spermatozoa isolated from all levels of the male tract were stable to hydrodynamic shear generated by vortexing, and underwent quantitative head-tail detachment in the presence of $n$ butylamine (Table 5). Rat epididymal spermatozoa were also stable to vortexing. $n$-Butylamine induced head detachment in $72 \%$ of rat caput epididymal spermatozoa whereas only $5-15 \%$ of the heads of those from the corpus and cauda epididymidis were detached. This low percentage 
Table 4. Head-tail detachment induced by $n$-butylamine after sodium cyanoborohydride reduction

\begin{tabular}{lccc}
\hline \multicolumn{1}{c}{ Spermatozoa } & $\begin{array}{c}\mathrm{NaCNBH}_{3} \\
\left(\mathrm{mg} / \mathrm{ml}^{3}\right.\end{array}$ & $\begin{array}{c}\text { No. of } \\
\text { exps }\end{array}$ & $\begin{array}{c}\text { Mean\% } \\
\text { detachment } \\
\text { (range) }\end{array}$ \\
\hline Rabbit (ejaculated) & - & 3 & 97 \\
& 10 & 3 & $74(71-80)$ \\
Rhesus monkey (ejaculated) & 20 & 3 & $26(23-49)$ \\
& 50 & 3 & $8(7-12)$ \\
Hamster (cauda epididymal) & - & 3 & $88(86-92)$ \\
Mouse (cauda epididymal) & 50 & 3 & $10(6-12)$ \\
$\quad$ (vas deferens) & 50 & 3 & $80(78-80)$ \\
& 50 & 2 & $10(10-11)$ \\
& - & 2 & $65(74-87)$ \\
& 50 & 2 & $66(65-75)$ \\
\end{tabular}

Sodium cyanoborohydride reduction was carried out with washed spermatozoa $\left(0.5-1 \times 10^{7}\right)$ in $1 \mathrm{ml} \mathrm{SEC} \mathrm{buffer}$ at $22^{\circ} \mathrm{C}$; control samples without $\mathrm{NaCNBH}_{3}$ reduction were used to determine background detachment.

Table 5. Head detachment of epididymal and vas deferens spermatozoa after vortexing in the presence or absence of $n$-butylamine

\begin{tabular}{ccc}
\hline Spermatozoa* & $\begin{array}{c}\text { With } n \text {-butylamine } \dagger \\
\text { (mean \%) }\end{array}$ & $\begin{array}{c}\text { Vortexing alone } \\
\text { (mean \% and range) }\end{array}$ \\
\hline Mouse Caput & 98 & $53(43-70)$ \\
Corpus & 93 & $37(29-45)$ \\
Cauda & 94 & $19(19-20)$ \\
Vas deferens & 92 & $5(1-9)$ \\
Rabbit Caput & 97 & $6(4-8)$ \\
Cauda & 98 & $4(3-4)$ \\
Vas deferens & 93 & $3(1-3)$ \\
Ejaculate & 95 & $3(1-5)$ \\
\hline
\end{tabular}

* $1-5 \times 10^{6}$ spermatozoa in $1 \mathrm{ml}$ SET buffer.

$\dagger 0 \cdot 1-0 \cdot 2 \mathrm{~mm}-n$-butylamine, $30 \mathrm{sec}$ vortexing.

$\ddagger$ Vortexing for $30 \mathrm{sec}$.

detachment may be due to sperm aggregation occurring after amine addition. The behaviour of mouse epididymal spermatozoa differed in that those isolated from the caput epididymidis were sensitive to hydromechanical shear generated by vortexing. However, the spermatozoa exhibited increasing stability to vortexing during passage through the epididymis to the vas deferens (Table 5). Electron microscopy showed that the majority of head-tail separations resulting from vortexing occurred at the same site as induced by primary amines. Lesser numbers of spermatozoa showed head separation between the basal plate and the capitulum of the tail, i.e. across the connecting filaments. These results demonstrate that there are species differences in the sensitivity towards hydromechanical shear of the sperm head-tail junction at the level of the nuclear envelope.

\section{Discussion}

The action of primary amines, aldehydes and thiols at specific sites within the sperm head-tail junction leads to the following concept of the biochemical linkages between (1) the nucleochromatin and the inner membrane of the nuclear envelope, (2) the inner and outer 
membranes of the nuclear envelope over the basal plate implantation fossa, and (3) between the basal plate(s) and the capitulum of the tail-neck complex. The inner membrane of the nuclear envelope is anchored by disulphide bonds to nuclear protein(s), possibly to one or more of the sulphydryl-rich basic chromatin proteins. The outer membrane of the nuclear envelope is firmly attached on its exterior face to the basal plate(s). The chemical nature of this attachment is unknown. Since high ionic strength buffers do not induce head-tail dissociation of rabbit or mouse spermatozoa (Millette et al., 1973), basal plate attachment is unlikely to be ionic, nor can it be by disulphide or aldimine bonds, as thiols and primary amines do not effect head-tail dissociation at this site. The inner and outer membranes of the nuclear envelope are bridged by periodic transverse structures along the entire implantation fossa, including the areas associated with the basal plate(s). These structures are absent after $n$-butylamine treatment and head-tail separation induced by this and other primary amines occurs between the inner and outer nuclear membranes. It is therefore likely that these periodic structures are the sites of the transimination reaction which allows headtail separation. The Schiff bases joining the inner and outer membranes of the nuclear envelope are probably located in the periodic transverse structures between these membranes. The intermolecular aldimine bond of these Schiff bases may link protein molecules within the periodic transverse structures or proteins of these structures and integral proteins of the nuclear envelope membranes. This cross-linking of sperm membrane proteins by aldimine bonds is another example of the joining of biological macromolecules by intermolecular linkages once thought to be unique to collagen and elastin (Diedrich \& Schnaitman, 1978; Starcher \& King, 1980; Leach, Rucker \& Van Dyke, 1981 ; Fujii \& Maruyama, 1982; Longas \& Meyer, 1982). The basal plate in turn is linked to the capitulum by connecting filaments. Although thiol reagents cleave sperm head and tail across these filaments it is unknown whether the disulphide bond(s) exist between proteins of the connecting filaments and those of the basal plate and capitulum or if the disulphide bonds are present within the filaments.

Primary amines are most effective in inducing sperm head-tail detachment at pH 10.5. Similar

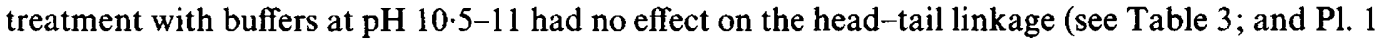
in Young \& Cooper, 1979). This result is consistent with the inability of secondary and tertiary amines, which cannot undergo a transimination reaction with $S$ chiff bases and yet have $\mathrm{p} K_{\mathrm{a}}$ values in the range $10-10 \cdot 5$, to induce head-tail detachment (Table 1). Even at $\mathrm{pH} 10 \cdot 5$, rabbit spermatozoa required about twice as much $n$-butylamine as mouse spermatozoa for quantitative head-tail detachment. Buffers at a higher $\mathrm{pH}(\mathrm{pH} 12.5)$ can effect sperm head-tail detachment. However, the reaction is much slower and the site of head detachment is different from that observed with primary amines (see also Millette et al., 1973). Therefore, sperm head-tail detachments that occur in the presence of primary amines are the result of a reaction of the amines with aldimine linkages within the head-tail junctional axis, and are not the consequence of the increase in $\mathrm{pH}$ which occurs when amine is added.

Formation of the basal plate, the connecting filaments, the capitulum and striated columns of the neck complex are early events in sperm differentiation which precede nuclear chromatin condensation and organization of the mid-and principal pieces of the tail. This early appearance of neck region structures includes the periodic cross-bridges (transverse electron densities) which appear to link the inner and outer membranes of the nuclear envelope in the region of basal plate contact with the spermatid nucleus (Fawcett \& Phillips, 1969). The distribution of these transverse bridges within the interspace between the nuclear membranes (Fawcett, 1975) may be related to the distribution of intramembranous particles revealed by freeze-fracture through the nuclear membranes overlying the basal plates (Friend \& Fawcett, 1974). In spite of the presence of ultrastructural cross bridges apparently linking the inner and outer membranes of the nuclear envelope, physical separation across this interspace can occur in some species, e.g. the mouse. Differences in susceptibility to mechanical shear across the nuclear membranes probably reflects species differences in the biochemical maturation of the neck region which occurs during epididymal sperm transport. For example, shear forces generated by vortexing are sufficient to 
detach the heads from the tails of mouse spermatozoa isolated from the caput epididymidis but those isolated from all parts of the rabbit epididymis are resistant to separation (Table 5). Cyanoborohydride reduction of mouse spermatozoa from the cauda epididymidis stabilized only about $12 \%$ of spermatozoa to the action of $n$-butylamine, while $48 \%$ of those from the vas deferens were so stabilized (Table 4). An inverse relationship therefore appears to exist between susceptibility to shear and the ability of cyanoborohydride to stabilize the mouse sperm head-tail junction to $n$-butylamine. This suggests that, in the mouse, aldimine bonds form during epididymal transit, and formation of the covalent links between the membranes of the nuclear envelope are not complete until these spermatozoa reach the vas deferens. The intermolecular aldimine bond thus appears to provide strength and rigidity to the head-tail junction and to stabilize it to shear by hydromechanical forces. Since rabbit spermatozoa are stable to these forces, formation of intermolecular Schiff bases linking the head and tail may be completed before spermiation in this species.

We thank Dr K. Sundaram for providing the rhesus monkey spermatozoa, and Ms K. Sweeny and Ms Miu-Ying Fong for their skilful technical assistance. This work was supported in part by the Rockefeller Foundation and NIH grants HD-10230 and HD-09215.

\section{References}

Balhorn, R., Gledhill, B.L. \& Wyrobeck, A.J. (1977) Mouse sperm chromatin proteins : quantitative isolation and partial characterization. Biochemistry, N.Y. 16, 4074-4080.

Brotherton, J. (1977) The splitting of sperm heads from tails in eight mammalian species and the measurement of their size. Andrologia 9, 1-14.

Calvin, H.I. (1976) Isolation and subfractionation of mammalian sperm heads and tails. In Methods in Cell Biology, Vol. 13, pp. 85-104. Ed. D. M. Prescott. Academic Press, New York.

Cooper, G.W. \& Young, R.J. (1977) Mammalian sperm are dissociated into heads and tails by primary amines: demonstration of intermolecular covalent bonds between the nuclear membranes and basal plate of the tail connecting piece. Anat. Rec. 187, 556, Abstr.

Dayagi, S. \& Degani, Y. (1970) Methods of formation of the carbon-nitrogen double bond. In The Chemistry of the Carbon-Nitrogen Double Bond, pp. 61-147. Ed. S. Patai. Interscience, New York.

Diedrich, D.L. \& Schnaitman, C.A. (1978) Lysyl-derived aldehydes in outer membrane proteins of Escherichia coli. Proc. natn. Acad. Sci. U.S.A. 75, 3708-3712.

Fawcett, D.W. (1975) The mammalian spermatozoon. Devl Biol. 44, 394-436.

Fawcett, D.W. \& Phillips, D.M. (1969) The fine structure and development of the neck region of the mammalian spermatozoon. Anat. Rec. 165, 153-183.

First, N.L. (1971) Collection and preservation of spermatozoa. In Methods in Mammalian Embryology, pp. 15-36. Ed. J. C. Daniel, Jr. Freeman, San Francisco.

Friend, D.S. \& Fawcett, D.W. (1974) Membrane differentiation in freeze-fractured mammalian sperm. $J$. Cell Biol. 63, 641-664.

Fujii, K. \& Maruyama, K. (1982) Existence of lysine- derived cross-linking in connectin, an elastic protein in muscle. Biochem. Biophys. Res. Commun. 104, 633640.

Lane, C.F. (1975) Sodium cyanoborohydride - a highly selective reducing agent for organic functional groups. Synthesis, pp. 135-146.

Leach, R.M., Rucker, R.B. \& Van Dyke, G.P. (1981) Egg shell membrane protein: a nonelastin desmosine/ isodesmosine-containing protein. Archs Biochem. Biophys. 207, 353-359.

Leussing, D.L. (1976) The formation of Schiff bases in the coordination sphere of metal ions. In Metal Ions in Biological Systems, Vol. 5, pp. 1-77. Ed. H. Siegel. Marcel Dekker, New York.

Longas, M.O. \& Meyer, K. (1982) Evidence that a reducible xylosyl-lysine is the protein linkage of dermatin sulfate. Proc. natn. Acad. Sci. U.S.A. 79, 6225-6228.

Millette, C.F., Spear, P.G., Gall, W.E. \& Edelman, G.M. (1973) Chemical dissection of mammalian spermatozoa. J. Cell Biol. 58, 662-675.

Starcher, B.C. \& King, G.S. (1980) The presence of desmosine and isodesmosine in eggshell membrane protein. Connect. Tiss. Res. 8, 53-55.

Young, R.J. (1979a) Rabbit sperm chromatin is decondensed by a thiol-induced proteolytic activity not endogenous to its nucleus. Biol. Reprod. 20, 10011004.

Young, R.J. (1979b) On the integrity of sperm DNA. Gamete Res. 2, 223-233.

Young, R.J. \& Cooper, G.W. (1979) Separation of the head and tail of mammalian spermatozoa by primary amines: evidence for their junction by Schiff bases. In The Spermatozoon, pp. 391-394. Eds D. W. Fawcett \& J. M. Bedford. Urban \& Schwarzenberg, Baltimore. 